\title{
DETERMINAÇÃO VOLUMETRICA DO BORO EM FERTILIZANTES. ESTUDOS SOBRE A QUANTIDADE DE MANITOL *
}

\author{
J. C. Alcarde ** \\ R. A. CATANI ** \\ O. S. SOUZA
}

\section{RESUMO}

\begin{abstract}
O presente trabalho teve como objetivo principal estudar a massa de manitol necessária para a titulação do ácido bórico com solução padronizada de $\mathrm{NaOH}$, a fim de aplicar os resultados obtidos na determinação volumétrica do boro, solúvel em ácido, em fertilizantes. Foram confeccionadas curvas de neutralização do $\mathrm{H}_{3} \mathrm{BO}_{3}$ pelo $\mathrm{NaOH}$, em soluções diluidas, na presença de quantidades variáveis de manitol e a referida titulação foi desenvolvida em função do $\mathrm{pH}$ do ponto final e da quantidade de manitol.

Os resultados obtidos permitiram verificar que a quantidade de manitol necessária na presente titulação é grandemente afetada pelo $\mathrm{pH}$ do ponto final e que há a possibilidade de se reduzir de $20,0 \mathrm{~g}$ para $5,0 \mathrm{~g}$ a quantidade de manitol usada na determinação do boro, solúvel em ácido, em fertilizantes, desde que se use o pH 8,0 para o ponto final da titulação e se proceda a eliminação do íon $\mathrm{NH}_{4}^{+}$
\end{abstract}

\section{INTRODUÇÃO}

A propriedade que o ácido bórico apresenta de aumentar a acidez de suas soluções aquosas, quando em presença de compostos poli-hidroxilados, foi descoberta em 1842 por Biot e o uso dessa propriedade para a determinação volumétrica do citado ácido foi feito por Thompson em 1893 (MELLON \& MORRIS, 1924; DEUSTCH \& OSOLING 1949; ROY, LAFERRIERE \& EDUARDS, 1957). Foram os numerosos estudos de Boeseken, em 1921 e 1922, que permitiram concluir que o aumento da acidez e da condutividade de soluções aquosas de ácido bórico, em presença de poliálcoois, fenois, hidro-

* Entregue para publicação em 3-1-1973.

** Departamnto de Química da Escola Superior de Agricultura "Luiz de Queiroz".

*** Bolsista da MANAH. 
xiácidos, ácidos cetônicos, açúcares, etc., é devido a formação de ácidos complexos mais fortes que os componentes originais (MELLON \& MORRIS, 1924). Em 1925 Herman demonstrou que compostos 1,2 e 1,3 diois, de configuração cis, geralmente podem formar dois tipos de complexos ácidos com o ácido bórico e presumiu que as estruturas dos mesmos seriam as representadas nas equações (1) e (2) (DEUSTCH \& OSOLING, 1949; ROY, LAFERRIERE \& EDUARDS, 1957).

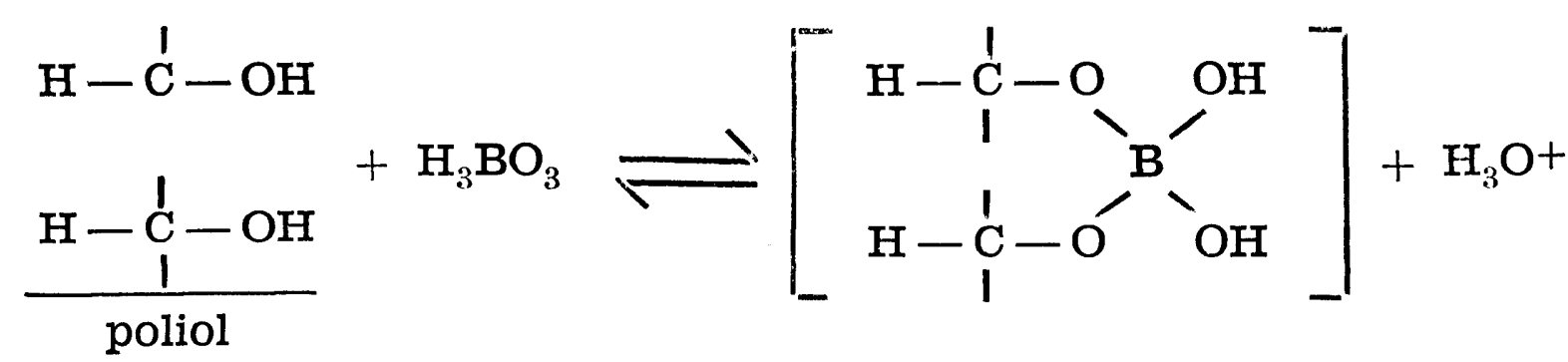

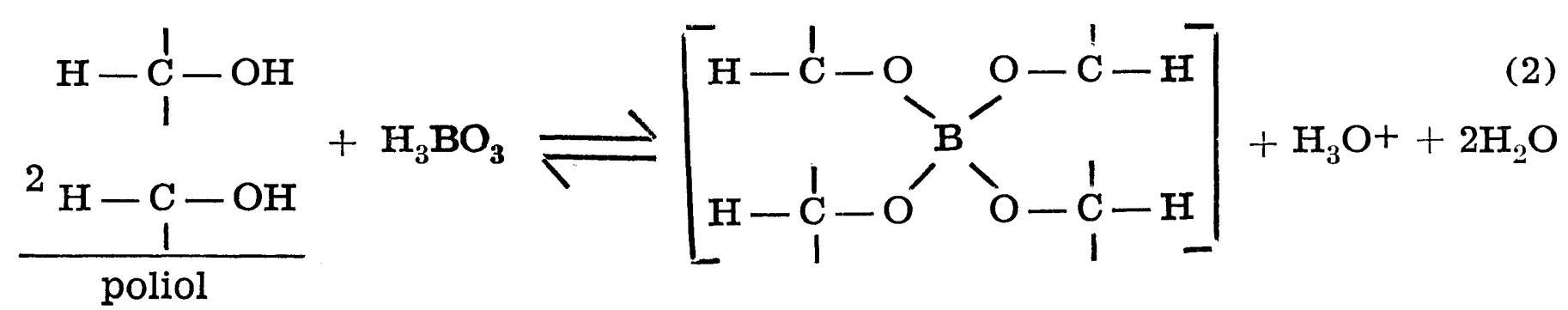

A eficiência dos diferentes poliois na complexação do ácido bórico é variável. MELLON \& MORRIS (1924) estudaram a ação de diversos polialcoois e açúcares na complexação do ácido bórico e concluiram que o manitol é o mais eficiente dos polialcoois e o açúcar invertido o mais eficiente dos açúcares. Realmente, o manitol é o poliol mais comumente usado na determinação volumétrica do ácido bórico.

Contudo, alguns detalhes importantes do equilíbrio que envolve a formação do complexo entre o ácido bórico e os poliois, inclusive o manitol, ainda não estão estabelecidos e continuam sendo objeto de estudos (DEUSTCH \& OSOLING, 1949; ROSS \& CATOTTI, 1949; ROY, LAFERRIERE \& EDUARDS, 1957; NICKERSON, 1968 e 1970; KNOECK \& TAYLOR, 1969; CAMPBELL, 1969). Embora a composição do complexo não esteja perfeitamente estabelecida no que diz respeito ao número de moléculas do poliol por molécula de $\mathrm{H}_{3} \mathrm{BO}_{3}$, é amplamente reconhecida as duas possibilidades sugeridas por Herman (1925) descritas nas equações (1) e (2) e que são representadas esquematicamente pelas equações (3), (4) e (5) (DEUSTCH \& OSOLING, 1949; ROSS \& CATTOTI 1949, NIES \& CAMPBELL, 1964; NOLLER, 1966; NICKERSON 1968 e 1970; CAMPBELL, 1969). 


$$
\begin{array}{r}
\mathrm{HB}+\mathrm{nP} \rightleftharpoons \mathrm{HBP}_{\mathrm{n}} \\
\mathrm{HBP}_{\mathrm{n}}+\mathrm{H}_{2} \mathrm{O} \rightleftharpoons \mathrm{BP}_{\mathrm{n}}-+\mathrm{H}_{3} \mathrm{O}^{+} \\
\mathrm{HB}+\mathrm{nP}+\mathrm{H}_{2} \mathrm{O} \rightleftharpoons \mathrm{PB}_{\mathrm{n}}-+\mathrm{H}_{3} \mathrm{O}^{+}
\end{array}
$$

em que HB representa o ácido bórico, $\mathrm{P}$ o poliol e $\mathrm{n}$ é um valor discutível, podendo ser 1 ou 2.

A titulação do ácido bórico com solução padronizada de $\mathrm{NaOH}$, na presença de manitol, constitue a base da determinação do boro em numerosos materiais (KOLTHOFF \& STENGER, 1947; HILLEBRAND \& OUTROS, 1953; FURMAN, 1962). E ainda prosseguem os estudos visando encontrar as condições mais apropriadas para a presente titulação (LE DUIGOU \& LAUER 1965; NAZARENKO \& ERMAK, 1968; BELCHER, 1970).

Um aspecto bastante controvertido na literatura diz respeito a quantidade de mantiol empregada na titulação do ácido bórico. MELLON' \& MORRIS (1924) estabeleceram a curva de neutralização de uma solução $0,1 \mathrm{~N}$ de $\mathrm{H}_{3} \mathrm{BO}_{3}$ por uma solução $0,2 \mathrm{~N}$ de $\mathrm{NaOH}$ empregando 4 moles do polialcool por mol de $\mathrm{H}_{3} \mathrm{BO}_{3}$. SCOTT (1939) recomenda $4 \mathrm{~g}$ e $8 \mathrm{~g}$ de manitol para determinação de boratos solúveis em água, empregando soluções $0,5 \mathrm{~N}$ e $1,0 \mathrm{~N}$ de $\mathrm{NaOH}$ respectivamente. KOLTHOFF \& STENGER (1947) relatam que são necessários $0,5 \mathrm{~g}$ a $0,7 \mathrm{~g}$ de manitol para titular $10 \mathrm{ml}$ de solução $0,1 \mathrm{~N}$ de $\mathrm{H}_{3} \mathrm{BO}_{3}$. E, em fertilizantes, materiais que contem relativamente pouco boro, às quantidades de manitol recomendadas para a determinação do citado elemento são $20 \mathrm{~g}$ (OFFICIAL METHODS OF ANALYSIS OF THE A.O.A.C. 1970) e $10 \mathrm{~g}$ (BORLAND, BROWNLIE \& GODDEN, 1967).

No entanto, ALCARDE, CATANI \& KROLL (1971) encontraram que $6,0 \mathrm{~g}$ de manitol são suficientes para titular 2,5 equivalentes miligramas ou milimoles de $\mathrm{H}_{3} \mathrm{BO}_{3}$, e o resultado desse estudo foi aplicado com sucesso na determinação do boro, solúvel em água, em fertilizantes.

Quanto a determinação do boro em fertilizantes é importante frisar que, na determinação do boro solúvel em água, a técnica de titulação empregada é a comum, sendo o ponto final indicado pela fenolftaleina ( $\mathrm{pH}$ aproximadamente 8,2 ). Já na determinação do boro solúvel em ácido a técnica de titulação empregada é a potenciométrica sendo usado para o ponto final o $\mathrm{pH} 6,30$. 
Em face dos equilibrios envolvidos na formação do complexo boro-manitol, constituiu objetivo do presente trabalho investigar a quantidade de manitol efetivamente necessária para titular uma certa quantidade de ácido bórico, levando em conta o $\mathrm{pH}$ do ponto final da titulação, a fim de aplicar os resultados na determinação do boro, solúvel em ácido, em fertilizantes.

\section{MÉTODOS}

\section{Reativos}

Dentre os reativos empregados merecem menção os seguintes:

Solução de $\mathrm{H}_{3} \mathrm{BO}_{3} \mathbf{0 , 1 0 0} \mathbf{~ N}$. Dissolver 3,0918 g de $\mathrm{H}_{3} \mathrm{BO}_{3}$ p.a. seco em estufa a $40^{\circ} \mathrm{C}$ por 4 horas, em água destilada, transferir para balão volumétrico de $500 \mathrm{ml}$ e completar o volume.

Solução de $\mathbf{N a O H}$ aproximadamente $0,025 \mathrm{~N}$, livre de $\mathrm{CO}_{2}$. Ferver 1 litro de água destilada por 20 minutos, com a finalidade de remover $\mathrm{CO}_{2}$, esfriar rapidamente e transferir para um frasco de plástico fechado com um dispositivo contendo "ascarite" para evitar a entrada do $\mathrm{CO}_{2}$ do ar. Juntar $2,5 \mathrm{ml}$ do sobrenadante de uma solução $10 \mathrm{~N}$ de $\mathrm{NaOH}$ (livre de $\mathrm{Na}_{2} \mathrm{CO}_{3}$ ) e agitar. Ligar esse frasco de plástico a uma bureta na qual também deve ser adaptado um dispositivo contendo "ascarite" para evitar a entrada de ar contendo $\mathrm{CO}_{2}$. Padronizá-la.

Solução de $\mathrm{NaOH}$ aproximadamente $0,05 \mathrm{~N}$, livre de $\mathrm{CO}_{2}$. Preparar conforme descrito para o preparo da solução $0,025 \mathrm{~N}$, somente que se deve diluir $5 \mathrm{ml}$ do sobrenadante da solução $10 \mathrm{~N}$ de $\mathrm{NaOH}$ (livre de $\mathrm{Na}_{2} \mathrm{CO}_{3}$ ). Padronizá-la.

Solução de $\mathbf{N a O H}$ aproxidamente $0,5 \mathrm{~N}$, livre de $\mathbf{C O}_{2}$. Preparar conforme descrito para o preparo da solução $0,025 \mathrm{~N}$, somente que se deve ferver 0,5 litro de água destilada e usar $25 \mathrm{ml}$ do sobrenadante da solução $10 \mathrm{~N}$ de $\mathrm{NaOH}$ (livre de $\mathrm{Na}_{2} \mathrm{CO}_{3}$ ).

Padronização das soluções aproximadamente $0,025 \mathrm{~N}$ e $0,05 \mathrm{~N}$ de $\mathrm{NaOH}$, livre de $\mathrm{CO}_{2}$.

Proceder conforme descrito no OFFICIAL METHODS OF ANALYSIS OF THE A.O.A.C. (1970).

\section{Métodos}

Determinação do $\mathrm{H}_{3} \mathrm{BO}_{3}$ pela técnica da titulação comum.

Este método constitue o fundamento da determinação volumé- 
trica do boro, solúvel em água, em fertilizantes (OFFICIAL METHODS OF ANALYSIS OF THE A.O.A.C., 1970).

1. Transferir $25 \mathrm{ml}$ de solução $0,100 \mathrm{~N}$ de $\mathrm{H}_{3} \mathrm{BO}_{3}$ para frasco de erlenmeyer de $300 \mathrm{ml}$ e juntar aproximadamente $150 \mathrm{ml}$ de água destilada.

2. Acrescentar $5-6$ gotas de solução de vermelho de metila a $0,5 \%$ e tornar a solução rósea pela adição de solução de $\mathrm{HCl}$ $(1+5)$.

3. Adicionar 3-4 "boillezers", cobrir com funil e ferver por 5 minutos para remover $\mathrm{CO}_{2}$. Esfriar em água enquanto coberto e lavar o funil e as paredes do frasco com pequena porção de água destilada.

4. Adicionar solução de $\mathrm{NaOH} 0,05 \mathrm{~N}$, livre de $\mathrm{CO}_{2}$, até obter-se a cor amarela do indicador.

5. Juntar manitol. No presente estudo foram usados 2, 4, 6, 10 e $20 \mathrm{~g}$ de manitol. Agitar até sua completa dissolução e acrescentar $1 \mathrm{ml}$ de solução de fenolftaleina a $1 \%$.

6. Titular com a solução padronizada $( \pm 0,05 \mathrm{~N})$ de $\mathrm{NaOH}$, livre de $\mathrm{CO}_{2}$, até a obtenção da cor rosada da fenolftaleina. Desenvolver uma prova em branco.

\section{Determinação do $\mathrm{H}_{3} \mathrm{BO}_{3}$ pela técnica da titulação potencio- métrica.}

Este método constitue o fundamento da determinação volumétrica do boro, solúvel em ácido, em fertilizantes (OFFICIAL METHODS OF ANALYSIS OF THE A.O.A.C., 1970).

1. Transferir $12,5 \mathrm{ml}$ de solução $0,100 \mathrm{~N}$ de $\mathrm{H}_{3} \mathrm{BO}_{3}$ para copo de $400 \mathrm{ml}$, adicionar $100 \mathrm{ml}$ de água destilada, $3 \mathrm{~g}$ de $\mathrm{NaCl}$ e 3 gotas de solução de vermelho de metila a $0,5 \%$.

2. Neutralizar a solução com solução de $\mathrm{NaOH} 0,5 \mathrm{~N}$ e reacidificar com solução de $\mathrm{HC1} 0,5 \mathrm{~N}$, adicionando 0,3 a $0,5 \mathrm{ml}$ de excesso.

4. Neutralizar grosseiramente a solução com solução $0,5 \mathrm{~N}$ de $\mathrm{NaOH}$, livre de $\mathrm{CO}_{2}$, e levar o copo para o conjunto de titulação que é constituido de um potenciômerto e um agitador magnético sob a bureta que contém a solução de $\mathrm{NaOH} \pm 0,025 \mathrm{~N}$, livre de $\mathrm{CO}_{2}$. Mergulhar o agitador e os eletrodos.

5. Ligar o agitador e o potenciômetro e ajustar o $\mathrm{pH}$ a exatamente 6,30 com solução de $\mathrm{NaOH} 0,025$, livre de $\mathrm{CO}_{2}$, ou $\mathrm{HC} 10,02 \mathrm{~N}$, conforme o caso. 
6. Adicionar manitol. No presente estudo foram usadas quantidades variáveis de manitol, conforme será adiante mostrado.

7. Titular com solução padronizada $( \pm 0,025 \mathrm{~N})$ de $\mathrm{NaOH}$, livre de $\mathrm{CO}_{2}$. Foram usados como ponto final os valores de $\mathrm{pH}$ $6,3-8,0$ e 8,5. Desenvolver uma prova em branco.

\section{RESULTADOS OBTIDOS E DISCUSSÃO}

Curvas de neutralização de uma solução diluida de $\mathrm{H}_{3} \mathrm{BO}_{3}$ por uma solução $0,025 \mathrm{~N}$ de $(\mathrm{NaOH}$, em função da quantidade de manitol.

Inicialmente procurou-se observar o comportamento da neutralização ácido bórico pelo $\mathrm{NaOH}$, em soluções diluidas, visto que MELLON \& MORRIS (1924) obtiveram as curvas dessa neutralização empregando soluções relativamente concentradas, isto é, solução $0,1 \mathrm{~N}$ de $\mathrm{H}_{3} \mathrm{BO}_{3}$ e $0,2 \mathrm{~N}$ de $\mathrm{NaOH}$.

Para tanto foram obtidas as curvas de neutralização de uma solução de ácido bórico contendo 1 milimol (ou miliequivalente) de $\mathrm{H}_{3} \mathrm{BO}_{3}$, por uma solução $0,025 \mathrm{~N}$ de $\mathrm{NaOH}$, em presença de quantidades variáveis de manitol $(20 \mathrm{~g}, 10 \mathrm{~g} \mathrm{e} 5 \mathrm{~g})$. As respectivas curvas estão graficamente representadas na figura 1.

Pode-se observar que a amplitude do intervalo de maior variação do $\mathrm{pH}$, nas proximidades do ponto de equivalência, diminue com o decréscimo da quantidade de manitol. Com $20 \mathrm{~g}$ de manitol esse intervalo está compreendido entre os valores de $\mathrm{pH} 7,0$ e 8,5, com $10 \mathrm{~g}$ de manitol entre os valores de $\mathrm{pH} 7,5$ e 8,5 e com $5 \mathrm{~g}$ de manitol entre os valores 7,75 e 8,5. É importante notar também que o valor de $\mathrm{pH}$ 6,30, recomendado para o ponto final da titulação na determinação do boro, solúvel em ácido, em fertilizantes (BORLAND, BROWNLIE \& GODDEN, 1967; OFFICIAL METHODS OF ANALYSIS OF THE A.O.A.C. 1970) está fora do intervalo de máxima variação de $\mathrm{pH}$. Consequentemente esses resultados permitem duvidar se no $\mathrm{pH}$ 6,30 o equilíbrio está completamente deslocado, isto é, se o $\mathrm{H}_{3} \mathrm{BO}_{3}$ foi quantitativamente titulado.

Titulação do ácido bórico em função da quantidade de manitol, pelas duas técnicas de titulação.

A titulação de uma solução padrão de ácido bórico com uma solução padronizada de $\mathrm{NaOH}$, em função da quantidade de manitol, pela técnica da titulação comum, foi feita por ALCARDE, 


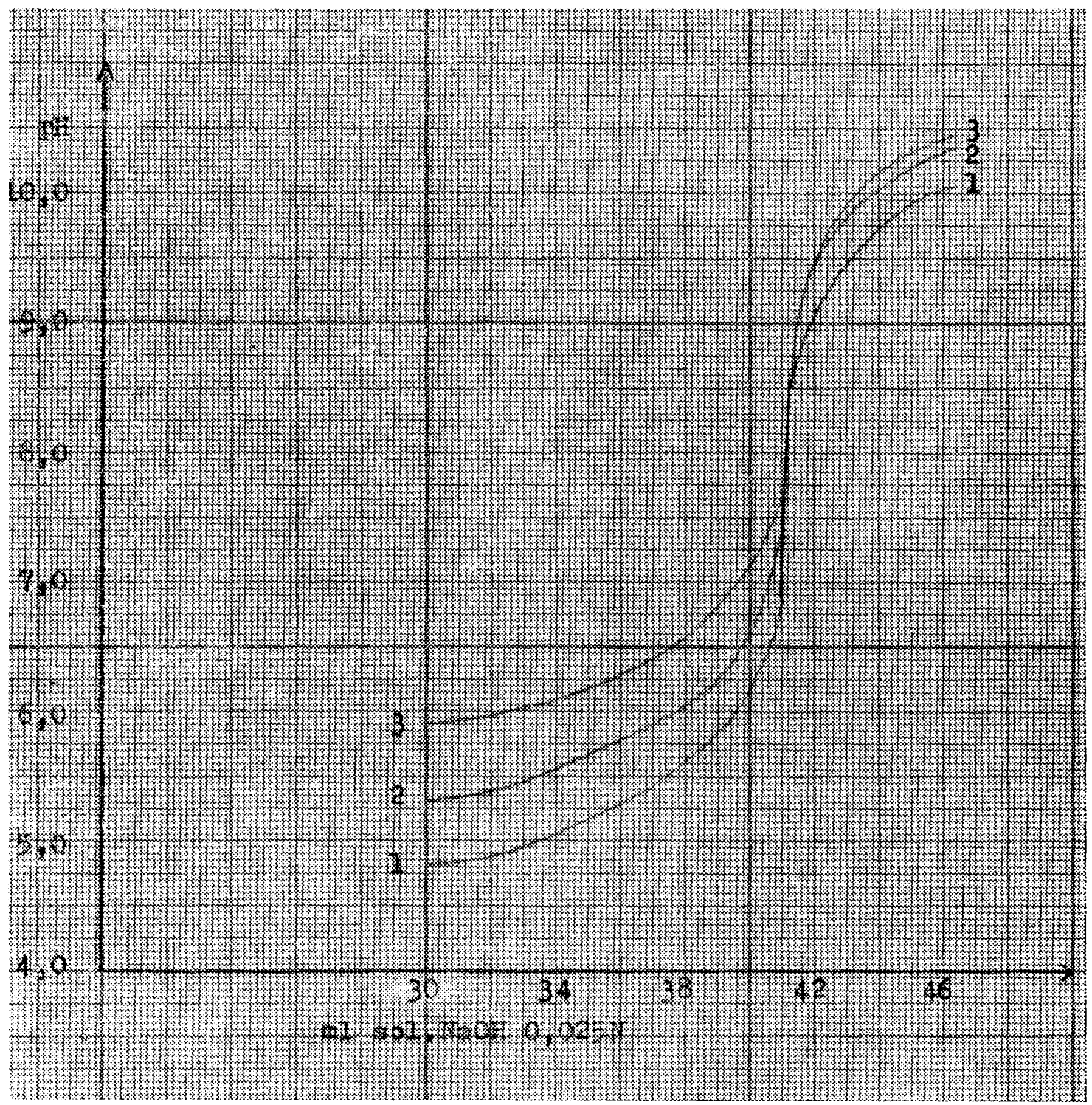

Figura 1. - Curvas de neutralização de uma solução de $\mathrm{H}_{3} \mathrm{BO}_{3}$, contendo 1 milimol (ou miliequivalente) de $\mathrm{H}_{3} \mathrm{BO}_{3}$, por uma solução $0,025 \mathrm{~N}$ de $\mathrm{NaOH}$, em função da quantidade de manitol. 1 - com $20 \mathrm{~g}$ de manitol; 2 - com $10 \mathrm{~g}$ de manitol; 3 - com $5 \mathrm{~g}$ de manitol.

CATANI \& KROLL (1971) e cujos resultados estão reproduzidos no quadro 1.

Os resultados da titulação de uma solução padrão de ácido bórico com uma solução padronizada de $\mathrm{NaOH}$, em função da quantidade de manitol, pela técnica da titulação potenciométrica e usando o pH 6,30 para o ponto final da titulação, conforme é recomendado na determinação do boro solúvel em ácido em fertilizantes (OFFICIAL METHODS OF ANALYSIS OF THE A.O.A.C. 1970), estão descritos no quadro 2 . 
Conforme é dado a observar nos quadro 1 e 2 , na técnica da QUADRO 1. - Resultados da titulação de $25 \mathrm{ml}$ de solução $0,100 \mathrm{~N}$ de $\mathrm{H}_{3} \mathrm{BO}_{3}$ com uma solução $0,0512 \mathrm{~N}$ de $\mathrm{NaOH}$, em função da quantidade de manitol, usando a técnica da titulação comum. Média de 3 repetições.

\begin{tabular}{cccc}
$\begin{array}{c}\text { Massa de } \\
\text { manitol usada } \\
(\mathrm{g})\end{array}$ & $\begin{array}{c}\mathrm{mg}_{3} \mathrm{BO}_{3} \\
\text { presente }\end{array}$ & $\begin{array}{c}\mathrm{ml} \mathrm{sol.} \mathrm{NaOH} \\
0,0512 \mathrm{~N} \text { gas- } \\
\text { tos na titul. }\end{array}$ & $\begin{array}{c}\mathrm{mg} \mathrm{H}_{3} \mathrm{BO}_{3} \\
\text { determ. }\end{array}$ \\
\hline 2 & 154,6 & 45,90 & 145.3 \\
4 & 154,6 & 48,45 & 153,4 \\
6 & 154,6 & 48,83 & 154,6 \\
10 & 154,6 & 48,82 & 154,6 \\
20 & 154,6 & 48,83 & 154,6 \\
\hline
\end{tabular}

QUADRO 2. - Resultados da titulação de $12,5 \mathrm{ml}$ de solução $0,100 \mathrm{~N}$ de $\mathrm{H}_{3} \mathrm{BO}_{3}$ com solução $0,0250 \mathrm{~N}$ de $\mathrm{NaOH}$, em função da quantidade de manitol, usando a técnica da titulação potenciométrica, com ponto final no $\mathrm{pH}-6,30$. Média de 3 repetições.

\begin{tabular}{cccc}
$\begin{array}{c}\text { Massa de } \\
\text { manitol usada } \\
(\mathrm{g})\end{array}$ & $\begin{array}{c}\mathrm{mg} \mathrm{H}_{3} \mathrm{BO}_{3} \\
\text { presente }\end{array}$ & $\begin{array}{c}\text { Presente ml de sol. } \\
\mathrm{NaOH} 0,0250 \mathrm{~N} \text { gasto } \\
\text { na titulação }\end{array}$ & $\begin{array}{c}\mathrm{mg} \mathrm{H}_{3} \mathrm{BO}_{3} \\
\text { determinados }\end{array}$ \\
\hline 2,0 & 77,3 & 35,60 & 53,9 \\
4,0 & 77,3 & 43,20 & 65,4 \\
6,0 & 77,3 & 47,40 & 73,3 \\
8,0 & 77,3 & 48,80 & 75,4 \\
10,0 & 77,3 & 48,70 & 75,3 \\
15,0 & 77,3 & 48,92 & 75,6 \\
20,0 & 77,3 & 49,94 & 77,2 \\
\hline
\end{tabular}

titulação comum são suficientes $6,0 \mathrm{~g}$ de manitol para titular 2,5 equivalentes miligramas ou milimoles de $\mathrm{H}_{3} \mathrm{BO}_{3}$, quantidade essa que mostrou resultados satisfatórios quando usada na determinação do boro, solúvel em água, em fertilizantes (ALCARDE, CATANI \& KROLL, 1971). Por outro lado, na técnica da titulação potenciométrica foram necessários $20,0 \mathrm{~g}$ de manitol para titular 1,25 equivalentes miligramas de manitol. 
Titulação do 'ácido bórico em função da quantidade manitol e do pH do ponto final, usando a técnica da titulação potenciométrica.

Uma diferença importante, do ponto de vista analítico, existente entre as titulações comum e potenciométrica usadas na determinação volumétrica do ácido bórico é o $\mathrm{pH}$ do ponto final da titulação. Enquanto que na titulação comum o ponto final é indicado pela fenolftaleina ( $\mathrm{pH}$ aproximadamente 8,2 ), na titulação potenciométrica o ponto final empregado situa-se no $\mathrm{pH}$ 6,30. Nesse fato parece residir a diferença encontrada na quantidade de manitol exigida nas duas técnicas de titulação. $\mathrm{E}$ isso parece evidente na equação que representa a formação do complexo boro-manitol (equação 5) onde se observa que o citado equilíbrio é influenciado diretamente pela concentração de manitol e pela concentração hidrogeniônica, as quais agem antagonicamente.

Tentando provar tal raciocínio, titulou-se o ácido bórico com solução aproximadamente $0,025 \mathrm{~N}$ de $\mathrm{NaOH}$, através da técnica da titulação potenciométrica, em função da quantidade de manitol e do $\mathrm{pH}$ do ponto final, conforme mostra o quadro 3, o qual contém também os resultados obtidos.

QUADRO 3. - Resultados da titulação de $12,5 \mathrm{ml}$ de solução $0,100 \mathrm{~N}$ de $\mathrm{H}_{3} \mathrm{BO}_{3}$ com solução $\pm 0,025 \mathrm{~N}$ de $\mathrm{NaOH}$, em função da quantidade de manitol e do $\mathrm{pH}$ do ponto final, através da técnica da titulação potenciométrica. Média de 3 repetições.

Massa de manitol $\mathrm{ml}$ de sol. de $\mathrm{NaOH} \pm 0,025 \mathrm{~N}$ gastos na titulação usada $(\mathrm{g})$

$$
\mathrm{pH} \text { final }=6,30 \mathrm{pH} \text { final }=8,00 \quad \mathrm{pH} \text { final }=8,50
$$

\begin{tabular}{rrrr}
\hline 20 & 50,0 & 50,6 & 50,8 \\
10 & 48,6 & 50,7 & 50,8 \\
7 & 46,7 & 50,5 & 50,7 \\
6 & 45,8 & 50,5 & 50,6 \\
5 & 44,0 & 50,5 & 50,7 \\
\hline
\end{tabular}

Esses resultados evidenciam que, usando o pH 8,0 ou 8,5 para o ponto final da titulação $5,0 \mathrm{~g}$ de manitol são suficientes para titular 1,25 equivalentes miligramas de $\mathrm{H}_{3} \mathrm{BO}_{3}$ e esclarecem também os dados contidos no quadro 1, obtidos pela titulação comum. Portanto existe a possibilidade de se reduzir a quantidade de manitol de $20 \mathrm{~g}$ para $5 \mathrm{~g}$ na determinação do boro, solúvel em ácido, em 
fertilizantes, empregando o pH 8,0 como ponto final da titulação. Contudo, a adoção desses valores na citada determinação acarreta a necessidade da eliminação do ion $\mathrm{NH}_{4}^{+}$que passa a interferir consumindo base.

\section{CONCLUSÓES}

Os resultados obtidos no presente trabalho possibilitaram as seguintes conclusões :

a) na titulação do ácido bórico com solução aproximadamente $0,025 \mathrm{~N}$ de $\mathrm{NaOH}$, o intervalo de máxima variação de $\mathrm{pH}$ varia de amplitude em função da quantidade de manitol presente. A amplitude diminue com o decréscimo da quantidade de manitol.

b) o valor de $\mathrm{pH}$ 6,30 adotado para o ponto final da titulação, na determinação volumétrica do boro solúvel em ácido em fertilizantes, está fora do intervalo da máxima variação de $\mathrm{pH}$. Isso permite duvidar se nesse $\mathrm{pH}$ todo o ácido bórico presente é complexado por $20 \mathrm{~g}$ de manitol, e, consequentemente, se o ácido é quantitativamente titulado.

c) a quantidade de manitol necessária para a determinação volumétrica do ácido bórico é grandemente influenciada pelo $\mathrm{pH}$ do ponto final da titulação.

d) para titular 1,25 milimoles de $\mathrm{H}_{3} \mathrm{BO}_{3}$ são suficientes $5 \mathrm{~g}$ de manitol, usando o pH 8,0 para o ponto final da titulação.

e) consequentemente, há a possibilidade de se reduzir a quantidade de manitol usada na determinação do boro, solúvel em ácido, em fertilizantes, de 20,0 para $5,0 \mathrm{~g}$, desde que se use o $\mathrm{pH} \mathrm{8,0} \mathrm{para}$ o ponto final da titulação e se proceda eliminação do ion $\mathrm{NH}_{4}^{+}$.

\section{SUMMARY}

VOLUMETRIC DETERMINATION OF BORON IN FERTILIZER STUDIES ON THE MANNITOL QUANTITY.

This paper deals on the studies of the mannitol quantity necessary for the titration of boric acid with standardized solution of $\mathrm{NaOH}$.

The mannitol quantity is influenced by the $\mathrm{pH}$ of the end point of the titration. The results allowed to conclude that there is the possibility to reduce from $20.0 \mathrm{~g}$ to $5.0 \mathrm{~g}$ the quantity of mannitol necessary for titration of boron soluble in acid in fertilizers, provided 
the $\mathrm{pH} 8$ be used as the end point of titration and proceeding the elimination of the ion $\mathrm{NH}_{4}^{+}$.

\section{LITERATURA CITADA}

ALCARDE, J. C., R. A. CATANI \& F. M. KROLL, 1971. Determinação do boro, solúvel em água, em fertilizantes. Anais da ESALQ, 28:287-296.

BELCHER, R., 1970. A comparative study of various complexing agents (polyols) used in the titration of boric acid. Anal. Chim. Acta, 50(2):261267.

BORLAND, H., I. A. BROWNLIE \& P. T. GODDEN, 1967. The determination of boron in fertilizers. Analyst, 92:47-53.

CAMPBELL Jr. G. W., 1969. Combining ratio of boric acid and alkali borate with mannitol. J. Inorg. Nucl. Chem. 31(8):2625-2627.

DEUSTCH, A. \& S. OSOLING, 1949. Conductrometric and potentiometric studies of the stoichiometry and equilibria of the boric acid - mannitol complexes. J. Am. Chem. Soc. 71(2):1637-1640.

FURMAN, N. H. (editor), 1962. Standard Methods of Chemical Analysis. Vol. I, 6th ed. D. Van Nostrand Co., Inc., Princeton, New Jersey. 1401 pp.

HILLEBRAND, W. F., G. E. F. LUNDELL, H. A. BRIGHT \& J. I. HOFFMAN, 1953. Applied Inorganic Analysis. John Willey \& Sons, Inc. New York, $1034 \mathrm{pp}$.

KNOECK, J. \& J. K. TAYLOR, 1969. Aqueous boric acid - borate - mannitol equilibria. Anal. Chem. 41(3):1730-1734.

KOLTHOFF, I. M. \& V. A. STENGER, 1947. Volumetric Analysis. Vol. II, 2nd ed. Interscience Publishers, Inc. New York. 374. pp.

LE DUIGOU, Y \& K. F. LAUVER, 1965. High - precison titrimetric of boric acid some calculations and measurements of inflection points. Anal. Chim. Acta, 33(2):222.

MELLON, M. G. \& V. N. MORRIS, 1924. An electrometric study of the titration of boric acid. Ind. and Eng. Chem. 16(2):123-126.

NAZARENKO, V. A. \& L. D. ERMAK, 1968. Alkalimetric determination of boric acid as polyhidroxylic complexes. Chem. Abstr. 69(8):32797 f.

NICKERSON, R. F., 1968. The combining ratio of boric acid and alkali borate with mannitol J. Inorg. Nucl. Chem., 30:1447-1455.

NICKERSON, R. F., 1970. On the composition of mannitol - borate complex. J. Inorg. Nucl. Chem. 32:1400-1402.

NIES, N. P. \& G. W. CAMPBELL, 1964. Inorganic Boron-Oxigen Chemistry. Em Boron, Metallo - Boron Compounds and Boranes, p. 53-231. Ed by R. M. Adams Interscience Publishers, New York.

NOLLER, C. R. 1966. Textbook of Organic Chemistry. 3rd ed., W. B. Saunders Co., Phyladelphia 760 pp.

OFFICIAL METHODS OF ANALYSIS OF THE A.O.A.C., 1970. 11th ed., p. 24-25. Published by Ass. of Off. Agr. Chem., Washington, D.C.

ROSS, S. D. \& A. J. CATOTTI, 1949. Equilibria of the boric acid-mannitol complexes. J. Am. Chem. Soc., 71(3):3563-3564. 
ROY, G. L., A. L. LAFERRIERE \& J. O. EDUARDS, 1957. A comparative study of polyol complexes of arsenite, borate, and tellurate ions. J. Inorg. Nucl. Chem. 4:106-114.

SCOTT, W. W., 1939. Scott's Standard Methods of Chemical Analysis 5th ed. Vol. I. Ed. by N. H. Furman., D. Van Nostrand Co., Inc., New York. 1234 pp. 